\title{
Relación entre roles administrativos y cultura organizacional en empresas del sector hotelero en las provincias de Soto y Guanentá \\ Relationship between administrative roles and organizational culture in the hotel sector companies at the provinces of Soto and Guanentá
}




\title{
Relación entre roles administrativos y cultura organizacional en empresas del sector hotelero en las provincias de Soto y Guanentá $^{1}$
}

\author{
Relationship between administrative roles and organizational \\ culture in the hotel sector companies at the provinces of Soto \\ and Guanentá
}

\section{Karolay Andrea Martínez-Serrano², Katherine Peña Guevara³ , Sebastián García- Méndez ${ }^{4}$,Yuly Andrea Ramírez-Sierra ${ }^{5}$.}

Artículo recibido en junio 29 de 2020; artículo aceptado en noviembre 13 de 2020

\begin{abstract}
Este artículo puede compartirse bajo la licencia Licencia Creative Commons Atribución-NoComercial-Compartir Igual 4.0 Internacional y se referencia usando el siguiente formato: Martínez-Serrano, K. A., Peña-Guevara, K. P., García-Méndez, S. y Ramírez-Sierra, Y. A. (2021). Relación entre roles administrativos y cultura organizacional en empresas del sector hotelero en las provincias de Soto y Guanentá. $I+D$ Revista de Investigaciones, 16(2), 18-25. https://doi.org/10.33304/revinv.v16n2-2021002.
\end{abstract}

\begin{abstract}
Resumen
Este artículo tiene como objetivo presentar la relación entre los roles administrativos y la cultura organizacional en empresas del sector hotelero de las provincias de Soto y Guanentá. Para tal fin se aplicaron instrumentos vinculables a los constructos objeto de estudio. Posteriormente, se realizó un análisis estadístico, utilizando para ello la correlación de Spearman, debido a las características de la normalidad. Los resultados más representativos indican que los roles informativos y decisorios tienen una relación representativa con la orientación al cambio. Esto se consolida como un aporte esencial en el entorno actual, teniendo en cuenta el cambiante comportamiento de los diversos sectores económicos, particularmente, el referido al turismo. De igual manera, se denota que los roles interpersonales promueven el cumplimiento de los objetivos y metas de las compañías.
\end{abstract}

Palabras clave: Cultura organizacional, estudio correlacional, roles administrativos, sector hotelero.

\footnotetext{
'Artículo de investigación, de tipo correlacional y corte transversal, con enfoque cuantitativo, resultado de un proyecto de investigación terminado, perteneciente al área de ciencias sociales e ingenierías, subárea Gestión Industrial, desarrollado en el Grupo de Investigación en productividad y gestión de Operaciones - SINERGIA, fue financiado por la Universidad de Investigación y Desarrollo (Bucaramanga, Colombia). Dirección: Calle 9 n. ${ }^{\circ}$ 23-55, PBX: (60) (7) 6352525. Fecha de inicio: agosto de 2019. Fecha de terminación: julio de 2020.

${ }^{2}$ Ingeniera Industrial, Universidad de Investigación y Desarrollo. Grupo de Investigación en productividad y gestión de Operaciones - SINERGIA, Universidad de Investigación y Desarrollo (Bucaramanga, Colombia). Dirección institucional: Calle 9 n. 23-55, PBX: (60) (7) 6352525. ORCID ID: https://orcid.org/0000-0002-2136-7122.Correo electrónico institucional: kmartinez5@udi.edu.co

${ }^{3}$ Ingeniera Industrial, Universidad de Investigación y Desarrollo (Bucaramanga, Colombia). Grupo de Investigación en productividad y gestión de Operaciones - SINERGIA, Universidad de Investigación y Desarrollo (Bucaramanga, Colombia). Dirección institucional: Calle 9 n. $23-55$, PBX: (60) (7) 6352525. ORCID ID: https://orcid.org/0000-0001-6380-3239. Correo electrónico institucional: kpena2@udi.edu.co

${ }^{4}$ Magíster en Administración, Universidad Santo Tomás. Docente investigador del Grupo de Investigación en productividad y gestión de Operaciones - SINERGIA, Universidad de Investigación y Desarrollo (Bucaramanga, Colombia) Dirección: Calle 9 n. ${ }^{\circ} 23-55$, PBX: (60) (7) 6352525. ORCID ID: https:// orcid.org/0000-0002-6077-0248. Correo electrónico institucional: sgarcia15@udi.edu.co

${ }^{5}$ Magíster en Ingeniería Industrial, Universidad Industrial de Santander. Docente investigador del Grupo de Investigación en productividad y gestión de Operaciones - SINERGIA, Universidad de Investigación y Desarrollo (Bucaramanga, Colombia) Dirección: Calle 9 n. ${ }^{\circ} 23-55$, PBX: (60) (7) 6352525. ORCID ID: https://orcid.org/0000-0001-6447-2291. Correo electrónico institucional: yramirez7@udi.edu.co
} 
Karolay Andrea Martínez-Serrano, Katherine Peña-Guevara, Sebastián García-Méndez, Yuly Andrea Ramírez-Sierra

Relación entre roles administrativos y cultura organizacional en empresas del sector hotelero en las provincias de Soto y Guanentá

\begin{abstract}
This article aims to present the relationship between administrative roles and organizational culture in the hotel sector companies at the provinces of Soto and Guanentá. For this reason, linkable instruments were applied to the object of study. Afterwards, a statistical analysis was made using Spearman's correlation method, due to the characteristics of normality. In that way, the most representative results indicate that information and decision-making roles have a representative relationship with change orientation. This consolidates as an essential contribution in the current environment, taking into account the changing behavior of the diverse economic sectors, particularly, the one referred to tourism. Therefore, it is noted that interpersonal roles, they promote the fulfillment of the companies' objectives and goals.
\end{abstract}

Keywords: Organizational culture, correlational study, administrative roles, hotel sector.

\section{Introducción}

Los roles administrativos son responsabilidades que asumen los gerentes o administradores con el objetivo de hacer cumplir las funciones de la empresa, en tal sentido, se destacan los roles interpersonales, informativos y decisorios, los cuales propenden por la consecución de las metas empresariales. En esta línea, la alta gerencia posee, administra y comparte la información con los colaboradores de la empresa, con el propósito de que las tareas queden claras y los fines sean alcanzados. Así mismo, son importantes la presencia de valores tales como el compromiso, la entrega y el buen trabajo en equipo, ya que impulsan beneficios organizativos.

En este contexto, se denota que el desempeño laboral de los trabajadores también es importante en el interior de las empresas, puesto que realizan funciones coordinadas con vistas al buen funcionamiento de las actividades y los esfuerzos compartidos en el marco de la entrega de resultados positivos para las metas temporales (Sarur Zanatta, 2013). De igual manera, la cultura organizacional, determina las técnicas, estructuras y sistemas objeto de ejecución por parte del personal de la compañía en el plano estratégico, táctico y operativo (Pedraza-Álvarez et al., 2015).

Los anteriores planteamientos permiten realizar un estudio en el sector hotelero con el objetivo de analizar la relación existente entre los roles administrativos y la cultura organizacional, particularmente en las Provincias de Soto y Guanentá. Esto, en últimas, tiene el propósito de formular acciones de mejora, ya que si los trabajadores internos realizan bien sus labores, se alcanzan las metas propuestas por las organizaciones. Por ese motivo, se hace necesario el estudio de variables asociadas al desempeño, trabajo y dirección de los trabajadores de las empresas hoteleras (Bracho, 2005).

\section{Marco teórico}

\section{Roles}

El rol administrativo se relaciona con el conjunto de funciones, normas y comportamientos por parte de una persona en un ámbito social o cultural, dicho de otra manera, es la labor ejercida por las personas en la sociedad; sin embargo, aún sigue la incógnita sobre qué es un administrador, un líder o un gerente, o si todos estos definitivamente desempeñan el mismo papel (Malavé y Piñango, 2012). Pacheco et al. (2017) citando a Mintzberg (2009) precisa que los roles de la alta gerencia y los comportamientos que se necesitan tener dentro de las empresas reflejan la importancia de la dedicación constante de un dirigente en una organización. Es significativo resaltar la eficiencia y la eficacia de los directivos, ya que estos últimos mejoran la calidad y dirigen a la empresa a la meta común.

En esta línea, Hellriegel (1998) postula la siguiente categorización en torno a los roles administrativos:

Roles interpersonales: Los roles interpersonales se clasifican en tres: I) el rol emblemático, en el cual el administrador representa a la organización, particularmente en temas referidos a las oportunidades de crecimiento y mejora del portafolio de productos y servicios; II) el rol de líder, que consiste en la dirección y coordinación de las actividades de los trabajadores, con el objetivo de que se cumplan las metas organizacionales; esto significa que el administrador es el ejemplo claro a seguir de todos los integrantes de la organización; III) el rol de enlace, en el que los administradores representan a las organizaciones tanto a nivel interno como externo, motivo por el cual les resulta importante la búsqueda y vinculación de personas que puedan influir positivamente en el éxito de la organización, dado sus conocimientos.

Roles informativos: Los roles informativos son tres: I) el rol de vigilancia, sumamente relevante dado que exige la búsqueda, recepción, selección y análisis de la información obtenida, la cual en su mayoría llega de forma oral, por lo que para una buena toma de decisiones, se debe identificar si es producto de rumores o si es verídica; II) el rol propagador, que caracteriza al administrador por la obtención de información y su difusión a las partes interesadas; III) el rol de vocero, el cual estriba en la transmisión de la información obtenida sobre la postura oficial de la organización, sea a todas las 
Karolay Andrea Martínez-Serrano, Katherine Peña-Guevara, Sebastián García-Méndez, Yuly Andrea Ramírez-Sierra Relación entre roles administrativos y cultura organizacional en empresas del sector hotelero en las provincias de Soto y Guanentá

personas de la empresa o aquellas que son ajenas, este último es importante, dado la exigencia de los medios por mayor información.

Roles de decisión: Los roles de decisiones se dividen en cuatro: I) el rol emprendedor, con el cual se buscan, crean y evalúan nuevos proyectos de mejora para la empresa, así como oportunidades para la organización; II) el rol de manejo de dificultades, visible cuando se deben tomar decisiones en posibles problemas y cambios que se presentan en la organización; III) el rol de asignación de recursos, que consiste en la destinación de los rubros, tales como dinero, equipo, personal y tiempo; IV) el rol de negociador, en el cual el administrador representa a la organización durante cualquier negociación.

\section{Método de medición de roles administrativos}

El método de medición de los roles administrativos es diverso. Para tal fin se precisa que en la presente investigación se utilizó el cuestionario propuesto por Fierro Cornejo (2015), el cual contempla las dimensiones interpersonales, informativas y decisorias.

\section{Cultura organizacional}

La cultura organizacional se relaciona con el conjunto de diversas creencias, valores, hábitos y tradiciones existentes en los diferentes grupos de las organizaciones, esto permite determinar cómo es el funcionamiento de una empresa, para lo cual se analizan las estrategias utilizadas, la estructura y sistema de cada organización. Es importante que una organización cuente con valores y normas para que cada una de las personas que la conforman estén identificados con ellos, tengan conductas positivas que incrementen la productividad $y$, como consecuencia, proyecten una imagen favorable de la empresa ante la sociedad. Algunos investigadores comparan la cultura organizacional con un iceberg, en el que la parte que se encuentra a la vista es la cultura, mientras que la parte oculta del iceberg representa los factores internos de las organizaciones (Arriola Miranda et al., 2011; Cantillo, 2013; Jaime Santana y Araujo Cabrera, 2007).

\section{Características}

Según Llanos et al. (2016), las características son las que diferencian la cultura organizacional de otras, entre ellas las más representativas son la cultura de identidad, la interacción, las personas, el control y el sistema abierto. En primer lugar, la cultura de identidad está determinada por las experiencias que los individuos de la organización han adquirido a través de los años y en cómo se adaptada a cada uno de ellos. Por eso, cuando los trabajadores ingresan a la empresa sin conocimiento detallado de su funcionamiento, con el tiempo aprenden de la cultura empresarial y el grado en que cada empleado se identifica con las labores y la cultura de los compañeros.
En segundo lugar, la interacción interviene en la cultura organizacional en que los empleados adquieren tratos con sus compañeros de trabajo, por ende, se mitigan las situaciones problemáticas y optimiza el rendimiento si existen buenas interacciones. La tercera característica describe a las personas, esta es fundamental para la compañía, pues las personas son parte esencial de ella, ya que las experiencias de cada individuo contribuyen a la creación y el mantenimiento de la cultura organizacional la cual se fortalece con el paso del tiempo.

Por su parte, el cuarto elemento hace referencia al control, este comprende el nivel de seguimiento de reglas que rigen a la compañía para que todo funcione de forma coordinada, ordenada e interdependiente. Finalmente, el quinto concepto, el sistema abierto, indica que es casi imposible que exista más de una cultura predominante en la empresa, aunque pueden existir subculturas entre los empleados. Por ese motivo, se debe optar por la existencia de una cultura global conocida y compartida por cada uno de los integrantes de la empresa, puesto que es importante que todos la reconozcan y aprueben.

\section{Tipos de cultura organizacional}

La cultura organizacional tiene una clasificación diversa de tipologías, por tanto, Harrison (1972), Ollarves (2006) y Valenzuela-Martínez (2015) precisan que la categorización depende de los objetivos empresariales y valores de las compañías, de modo que sugieren cuatro tipos de orientaciones:

- Organizaciones orientadas al poder: Las empresas que conforman esta tipología tienen como objetivo primordial la competitividad empresarial (García, Santana y Guzmán, 2020), pues los valores están orientados a fortalecer la posición en el mercado. Por lo tanto, suelen tomar decisiones mediante un directivo con autonomía y suficiente control sobre cada uno de los empleados.

- Organizaciones orientadas a la norma: Se encuentran empresas enfocadas en garantizar su estabilidad y seguridad, basadas en el cumplimiento riguroso de las normas y responsabilidades que cada persona tiene en el puesto de trabajo, estas buscan garantizar el correcto funcionamiento del proceso y procedimiento llevados a cabo en la organización.

- Organizaciones orientadas a los resultados: Son aquellas que se centran en la eficacia del desarrollo de los procesos que realizan, la visión se guía a la reducción de costes y el cumplimiento de cada uno de los objetivos empresariales en el menor tiempo posible, pero con la mayor eficacia que pueda generar. Las organizaciones que componen esta tipología tienden a ser más competitivas en el entorno empresarial. 
Karolay Andrea Martínez-Serrano, Katherine Peña-Guevara, Sebastián García-Méndez, Yuly Andrea Ramírez-Sierra

Relación entre roles administrativos y cultura organizacional en empresas del sector hotelero en las provincias de Soto y Guanentá

- Organizaciones orientadas a las personas: Son las empresas que buscan la realización personal de los empleados, incentivándolos por medio de la formación, motivación, creatividad y satisfacción. En las organizaciones orientadas a las personas se tiene como prioridad a los miembros, por ende, tienden a darles apoyo. Actualmente, las organizaciones se inclinan más por el modelo orientado a las personas y los resultados, en cambio evitan las culturas estrictas y guiadas según las normas.

\section{Instrumentos de medición de la Cultura Organi- zacional}

La medición de la cultura organizacional es muy diversa, particularmente la presente investigación toma como referente el utilizado por Contreras Cueva y Gómez Gómez (2018), que se caracteriza por ser un modelo de gran importancia en el plano empresarial, esto valida su alto uso por parte de la comunidad científica (Ali Ahmady et al., 2016; Denison et al., 2004; Denison y Spreitzer, 1991).

\section{Correlación de Spearman}

El coeficiente de correlación de Spearman es una medida de relación lineal, este utiliza los datos suministrados con anterioridad para emplear en un campo multivariado, cuyo propósito es establecer las similitudes o diferencias entre las variables de estudio, así consigue medir el grado de relación entre dos variables aleatorias (Santander y Ruiz, 2004).

\section{Metodología}

\section{Tipo de estudio}

La investigación es de enfoque cuantitativo. El estudio se basó en un diseño no experimental de corte transaccional o transversal en vista de su realización en un determinado momento y una sola vez. Además, el alcance es descriptivo debido a que permite identificar las propiedades y características importantes de las variables a estudiar. Adicionalmente, se denota un alcance descriptivo-correlacional, teniendo en cuenta que primero se describen los roles administrativos y la cultura organizacional, y posteriormente se realiza la correlación entre las variables objeto de estudio.

\section{Participantes}

La población objeto de estudio en la investigación involucra a 58 empresas hoteleras afiliadas a Cotelco. En tal sentido, se utilizó un muestreo no probabilístico a conveniencia, en el marco de definir un tamaño muestral de 39 unidades empresariales. Los instrumentos fueron aplicados a 39 Gerentes-Administradores (17 hombres y 22 mujeres) y a 45 trabajadores (16 hombres y 16 mujeres). En la investigación se realizó la aplicación del instrumento una única vez en cada uno de los elementos de la población, con el fin de identificar la relación entre las variables objeto de estudio.

\section{Materiales e instrumentos}

Los instrumentos de recolección de información utilizados en el estudio sobre los roles administrativos y la cultura organizacional, fueron los referenciados por Bonavia et al. (2009), Contreras Cueva y Gómez Gómez (2018) y Fierro Cornejo (2015), los cuales han sido adoptados en diferentes investigaciones a nivel nacional e internacional.

El instrumento de Fierro Cornejo (2015) contempla una escala Likert de 5 niveles -Muy en desacuerdo, En desacuerdo, Indeciso, De acuerdo, Muy de acuerdoestá integrado por 24 preguntas y cuenta con un alfa de Cronbach de 0,92. Por su parte, el instrumento de Contreras Cueva y Gómez Gómez (2018) utiliza la escala Likert de 5 niveles: Nunca, Casi nunca, A veces, Con frecuencia y Siempre; está conformado por 60 ítems y cuenta con un alfa de Cronbach de 0,90. Lo anterior indica la confiabilidad de los instrumentos.

\section{Procedimiento}

Para el estudio en mención se realiza la medición de los constructos en los establecimientos hoteleros, posteriormente se determina la relación por medio de la correlación de Spearman, teniendo en cuenta las características de normalidad.

\section{Resultados}

En las secciones que siguen se analizan y presentan los resultados de la medición de las variables, que integran los roles administrativos y la cultura organizacional, así como sus relaciones identificadas mediante los coeficientes de correlación de Spearman.

\section{Roles administrativos}

Los roles administrativos se relacionan con el conjunto de funciones, normas y comportamientos por parte de una persona en un ámbito social o cultural, lo que implica un grado significativo de responsabilidad con los trabajadores y la organización, con el fin de lograr la mejora continua y las buenas relaciones laborales. En la Tabla 1 se presentan los resultados del instrumento de Fierro Cornejo (2015) aplicado a los gerentes y administradores de las unidades empresariales en las provincias de Soto y Guanentá.

Tabla 1.

Roles administrativos

\begin{tabular}{cc}
\hline Tipos de roles & Puntaje \\
\hline Interpersonales (R1) & 4,65 \\
Informativos (R2) & 4,62
\end{tabular}


Karolay Andrea Martínez-Serrano, Katherine Peña-Guevara, Sebastián García-Méndez, Yuly Andrea Ramírez-Sierra Relación entre roles administrativos y cultura organizacional en empresas del sector hotelero en las provincias de Soto y Guanentá

\begin{tabular}{cc}
\hline Tipos de roles & Puntaje \\
\hline Decisorios (R3) & 4,74 \\
\hline
\end{tabular}

Fuente: Autores.

Es importante resaltar que el puntaje más alto es 5 y el más bajo 1 . Se observa en la Tabla 1 que cada rol obtuvo los siguientes puntajes: interpersonales, 4,65; informativos, 4,62; decisorios, 4,74, siendo este último el más alto. En consecuencia, se infiere que las empresas hoteleras hacen esfuerzos para lograr altas posiciones con los recursos que poseen, pues lo importante para los gerentes y administradores es lograr la mejora continua de la empresa y alcanzar los objetivos propuestos.

En tanto, la calificación baja del rol informativo permite inferir que la forma de planear y organizar acciones de mejora de la empresa varía un poco, porque el uso de los equipos tecnológicos y los sistemas de información no poseen la atención que requieren. Aquellos elementos son clave para el buen funcionamiento, ya que son las herramientas estratégicas que permiten analizar y hacer seguimiento de los beneficios que se deben tener en las organizaciones para lograr una administración adecuada.

\section{Cultura organizacional}

La cultura organizacional considera el tipo de estrategias utilizadas, las estructuras y los sistemas más importantes que una organización debe tener, es relevante resaltar los valores y normas de cada persona que conforma la organización para mantener una calidad de vida laboral satisfactoria. En este aspecto, los resultados se postulan en la Tabla 2.

Tabla 2.

Cultura Organizacional

\begin{tabular}{cc}
\hline Tipos de cultura & Puntaje \\
\hline Toma de decisión (C1) & 4,54 \\
Trabajo en equipo (C2) & 4,5 \\
Desarrollo de capacidades (C3) & 3,85 \\
Valores (C4) & 4,28 \\
Acuerdos (C5) & 4,11 \\
Coordinación e integración (C6) & 4,3 \\
Orientación al cambio (C7) & 4,07 \\
Orientación al cliente (C8) & 4,04 \\
Aprendizaje organizativo (C9) & 4,14 \\
Dirección y propósitos estratégicos (C10) & 4,19
\end{tabular}

\begin{tabular}{cc} 
Metas y objetivos (C11) & 4,55 \\
Visión (C12) & 4,48 \\
\hline
\end{tabular}

Fuente: Autores.

Las ponderaciones obtenidas permiten concluir que la dimensión que alcanzó una calificación superior fue la relacionada con metas y objetivos (4,55 puntos), lo que conlleva a inferir que los hoteles tienen un liderazgo visionario, es decir con miras al futuro, ya que se evaluaron escenarios como el cumplimiento de objetivos y el compromiso de los trabajadores en el logro de sus metas.

Por otra parte, el desarrollo de las capacidades obtuvo una puntuación de 3,85 , siendo esta la más baja, por consiguiente, se concluye que algunas organizaciones tienen poco interés en desarrollar y explotar las capacidades y habilidades de sus empleados. De ahí, se evidencia la carencia de actividades o programas que permitan reconocer a los trabajadores como una ventaja competitiva.

De igual manera, se muestra que la dimensión correspondiente a la orientación al cliente tiene una puntuación de 4,04, lo que indica que podría existir una falta de concentración de iniciativas hacia la satisfacción de los clientes y poco interés en las recomendaciones y/o comentarios que estos hacen sobre las empresas. Cabe resaltar que esta dimensión en el marco del sector servicios es una de las más importantes, ya que los clientes tienen la capacidad de evidenciar el valor agregado de los servicios y son interesados que tienen alto impacto en el fortalecimiento de los hoteles.

\section{Correlación de Spearman}

La correlación de las variables se realizó por medio del coeficiente de Rho de Spearman, teniendo en cuenta los resultados presentados en la prueba de normalidad. La Tabla 3 presenta la correlación de las variables de roles administrativos y cultura organizacional, esta permite observar que las tres dimensiones de las primeras variables se encuentran correlacionadas con cuatro de las doce categorías de la segunda variable (cultura organizacional).

Tabla 3.

Correlación de Spearman variables de roles administrativos y cultura organizacional

\begin{tabular}{|c|c|c|c|c|c|c|c|c|c|c|c|c|}
\hline & C1 & C2 & C3 & C4 & C5 & C6 & C7 & C8 & C9 & C10 & C11 & C12 \\
\hline R1 & 0,22 & 0,11 & 0,17 & 0,19 & $-0,25$ & 0,15 & 0,24 & $-0,20$ & 0,16 & 0,25 & 0,24 & 0,10 \\
\hline $\mathrm{R} 2$ & 0,23 & 0,11 & 0,08 & 0,12 & $-0,15$ & 0,12 & 0,31 & $-0,10$ & $-0,07$ & 0,12 & 0,26 & 0,18 \\
\hline R3 & 0,21 & $-0,02$ & 0,15 & 0,00 & $-0,15$ & $-0,02$ & 0,30 & 0,10 & $-0,10$ & 0,03 & 0,08 & 0,09 \\
\hline
\end{tabular}

Fuente: Autores. Nota: La codificación de las variables es la siguiente: Roles interpersonales (R1), Roles informativos (R2), Roles de decisión (R3), Toma de decisiones (C1), Trabajo en equipo (C2), Desarrollo de las capacidades (C3), Valores (C4), Acuerdos (C5), Coordinación e integración (C6), Orientación al cambio (C7), Orientación al cliente (C8), Aprendizaje organizativo (C9), Dirección y propósitos estratégicos (C10), Metas y objetivos (C11), Visión (C12). * La correlación es significativa en el nivel 0,05 (bilateral). ** La correlación es significativa en el nivel 0,01 (bilateral). 
Karolay Andrea Martínez-Serrano, Katherine Peña-Guevara, Sebastián García-Méndez, Yuly Andrea Ramírez-Sierra

Relación entre roles administrativos y cultura organizacional en empresas del sector hotelero en las provincias de Soto y Guanentá

A partir de los resultados de la Tabla 3 se identifica que no existe una relación significativa entre las variables de roles administrativos y cultura organizacional en los establecimientos del sector hotelero de las provincias de Soto y Guanentá. No obstante, en las compañías descritas, se visualizan elementos a mejorar por medio de la gestión empresarial, tales como el proceso de toma decisiones y la difusión de la información a las partes interesadas, ya que generan elementos a favor de la orientación al cambio, la cual actualmente se constituye como un insumo de ventaja competitiva en el marco de una economía con alto porcentaje de incertidumbre. Así mismo, se reconoce que el componente interpersonal propicia un escenario promisorio para el logro de las metas y objetivos de la entidad. En otros términos, la sinergia y cohesión de las diversas áreas funcionales se genera por medio de la acción conjunta y complementaria de diversos tipos de individuos, cada uno de ellos con sus virtudes y cualidades.

Por otra parte, se observa que los roles administrativos no generan ningún efecto positivo en los acuerdos con los diversos integrantes de la organización, por lo que se deberán analizar diferentes elementos anexos a la gestión del talento humano, con el propósito de propiciar entornos de trabajo coherentes y amenos para la totalidad de los colaboradores del sector hotelero.

\section{Conclusiones}

Las empresas hoteleras de las provincias de Soto y Guanentá poseen oportunidades de crecimiento debido al compromiso que tienen los gerentes y administradores. En ese sentido, con el fin de lograr una buena toma de decisiones en un hotel, son necesarios el adecuado uso de la tecnología, el seguimiento de las políticas establecidas para garantizar procedimientos de manera coordinada e informada $y$, sobre todo, una buena utilización de todos los recursos de la empresa. Sin desconocer que el personal administrativo fomenta el éxito de las empresas, y eleva los índices de oportunidades laborales y de servicios en el sector, cuando trabaja en equipo.

Así mismo, es imprescindible vislumbrar la posibilidad de establecer vínculos con entidades gubernamentales para realizar análisis holísticos del sistema turístico del territorio (García et al. 2020).

Los roles administrativos obtuvieron resultados favorables, lo cual indica que las empresas hoteleras, principalmente de las provincias de Guanentá, consideran a los integrantes de la organización como parte fundamental para el desarrollo de la misma, razón por la que los trabajadores se sienten muy satisfechos con la realización de las labores, debido a que la entidad cumple con las expectativas. Lo anterior permite el buen desempeño de las actividades diarias para el cumplimiento de los objetivos de la empresa en los planos temporales especificados.
Los resultados sobre la cultura organizacional de los establecimientos hoteleros de la provincia de Soto y Guanentá indican que cuando los trabajadores ingresan a la empresa no conocen el funcionamiento con detalle, sin embargo, los lineamientos brindados por los administradores posibilitan una mayor alineación con la tarea encomendada, indicio de que la cultura se encuentra orientada al aprendizaje. Otro aspecto relevante es que los miembros de las organizaciones se caracterizan por unos valores y creencias personales pero, en función de evitar conflictos laborales con los directivos u otros miembros de la empresa, estos optan por la existencia de una cultura global que contemple a todas las partes interesadas.

El departamento de Santander tiene grandes orientaciones turísticas, por consiguiente, se requiere que los establecimientos hoteleros, particularmente los presentes en la provincia de Soto y Guanentá, desarrollen procesos de mejora en torno a la satisfacción laboral, la solución de conflictos, la gestión del cambio, el manejo de la información y la administración hotelera; entre otros aspectos, los cuales permitirán el desarrollo de capacidades que son insumo de ventaja competitiva.

Por último, la investigación en mención establece como futuras líneas de investigación el rol del liderazgo, la comunicación organizacional y el proceso de toma de decisiones en la cultura organizacional de los establecimientos hoteleros, estos serán potencialmente objeto de estudio de los presentes investigadores e interesados en el sector del turismo, que se reconoce como un campo importante en la sociedad actual.

\section{Referencias}

Ali Ahmady, G., Nikooravesh, A., y Mehrpour, M. (2016). Effect of Organizational Culture on knowledge Management Based on Denison Model. $3^{\text {rd }}$ International Conference on New Challenges in Management and Organization: Organization and Leadership, 230, 387-395. https://www.scienc edirect.com/journal/procedia-social-and-behavio $\mathrm{ral}$-sciences/vol/230/suppl/C

Arriola Miranda, M. A., Salas Rivera, É., y Bernabé González, T. B. (2011). El clima como manifestación objetiva de la cultura organizacional. Revista Ciencias Estratégicas, 9(25), 109-127. https://www. redalyc.org/articulo.oa?id=151322413008

Bonavia, T., Prado, V., y Barberá, D. (2009). Spanish Adaptation and Factor Structure of the "Denison Organizational Culture Survey». Psicothema, 21(4), 633-638.

Bracho, A. C. (2005). Desempeño gerencial: funciones y roles en la practica. Compendium, 8(14), 5-19. https://www.redalyc.org/articulo.oa?id=88001402 
Karolay Andrea Martínez-Serrano, Katherine Peña-Guevara, Sebastián García-Méndez, Yuly Andrea Ramírez-Sierra Relación entre roles administrativos y cultura organizacional en empresas del sector hotelero en las provincias de Soto y Guanentá

Cantillo, J.C. (2013). Incidencia de la cultura organizacional en el desempeño. Revista Económicas CUC, 34(1), 131-152. https://revistascientificas.cuc.edu.co/econ omicascuc/article/view/580

Contreras Cueva, A. B., y Gómez Gómez, A. (2018). Aplicación de los Cuestionarios de Denison para determinar las características de la Cultura Organizacional. Revista de Investigación Sigma, 5(1), 59-86. http://dx.doi.org/10.24133/sigma.v5i01.1204

Denison, D., Lief, C., y Ward, J. L. (2004). Culture in FamilyOwned Enterprises: Recognizing and Leveraging Unique Strengths. Family Business Review, 17(1), 61-70. https://doi.org/10.1111/j.1741-6248.2004.000 04.x

Denison, D., y Spreitzer, G. M. (1991). Organizational culture and organizational development: a competing values approach. Research in Change and Organizational Development (pp. 1-21). JAI Press.

Fierro Cornejo, Z. G. (2015). Roles gerenciales del personal directivo y la eficiencia laboral del personal administrativo en la Municipalidad Provincial de Huancavelica - Año 2012 [Tesis de Licenciatura, Universidad Nacional Huancavelica]. http://reposi to rio.unh.edu.pe/handle/UNH/81

García, A. M., Santana, C. M., y Guzmán, A.P. (2020). Modelo de la Gestión del Talento Humano como desarrollo competitivo y sostenible, sector de Turismo en Santander, Colombia. I+D Revista de Investigaciones, 15(2), 107-118. https://doi.org/10.33304/revinv.v15n 2-2020010

García, S., Álvarez, L. M., y Peñuela, L. T. (2020). Diseño de un plan estratégico de turismo en el municipio de Guadalupe (Santander), Innovando desde las Organizaciones (pp. 241-255). Unisangil Editora.

Harrison, R. (1972). Understanding your organisation's character. Harvard Business Review.

Hellriegel, D. (1998). Administración. (7ª ed.) International Thomson Editores.

Jaime Santana, P., y Araujo Cabrera, Y. (2007). Clima y cultura organizacional: ¿dos constructos para explicar un mismo fenómeno? Decisiones basadas en el conocimiento y en el papel social de la empresa: XX Congreso anual de AEDEM, 1, 296-324.https:// dialnet.unirioja.es/servlet/libro?codigo $=275448$

Llanos, M., Pacheco, M. G., Romero, E. M., Coello, F., y Armas, Y. M. (2016). La cultura organizacional: eje de acción de la gestión humana. Universidad ECOTEC.
Malavé, J., y Piñango, R. (2012). Líderes, gerentes y emprendedores: un estudio empírico de actitudes y significados. Revista Venezolana de Gerencia, 17(60), 676-694. https://doi.org/10.31876/revista. v17i60.10938

Ollarves, Y. (2006). Cultura Organizacional y propiedades motivantes del puesto de trabajo en una Institución de Educación Superior. Investigación y Postgrado, 21(1), 125-151. https://www.redalyc.org/articulo.o a?i d=65821106

Pacheco, Y., Molina, Y., y Arévalo, J. (2017). Los roles gerenciales de Mintzberg: una evidencia empírica en la universidad. I+D Revista de Investigaciones, 10(2), 102-111. https://doi.org/10.33304/revinv.v10n 2-2017009

Pedraza-Álvarez, L., Obispo-Salazar, K., VásquezGonzález, L., y Gómez-Gómez, L. (2015). Cultura organizacional desde la teoría de Edgar Schein: Estudio fenomenológico. Clío América, 9(17), 17-25. https://doi.org/10.21676/23897848.1462

Santander, A., y Ruiz, R. (2004). Relación entre variables cuantitativas. ECIMED.

Sarur Zanatta, M. S. (2013). La importancia del capital intelectual en las Organizaciones. Ciencia Administrativa, (1), 39-45. https://www.uv.mx/iiesca/ files/2014/01/05CA201301.pdf

Valenzuela-Martínez, P. A. (2015). Incidencia de la Cultura Organizacional en la Participación de Instituciones Educativas en Certámenes de Calidad. Revista Ciencia, Ingeniería y Desarrollo Tec Lerdo, 1(1), 262-267. https://docplayer.es/23673346-.html. 